\title{
Perfil gerencial en los sectores productivos de la provincia de Tungurahua
}

\section{Management profile in the productive sectors of the province of Tungurahua}

\author{
Mg. Carlos Mejía-Vayas ${ }^{1}$ \\ carlosvmejia@uta.edu.ec \\ Mg. Jorge Jordán-Vaca \\ jorgeejordan@uta.edu.ec \\ Mg. Leonardo Ballesteros-López ${ }^{3}$ \\ jorgeejordan@uta.edu.ec \\ Mg. José Herrera-Herrera \\ josebherrera@uta.edu.ec \\ Mg. César Guerrero-Velástegui ${ }^{5}$ \\ ca.guerrero@uta.edu.ec \\ Ing. Carlos Masaquiza-Caiza \\ cmasaquiza@bhconsultores.com
}

Recibido: 1/09/2017, Aceptado: 1/11/2017

\section{RESUMEN}

El propósito de este estudio es realizar un análisis prospectivo que facilita la construcción y desarrollo de las habilidades gerenciales a través de un modelo que refleja un perfil por competencias gerenciales para los gerentes de los sectores productivos de la provincia de Tungurahua en el contexto de la mejora continua y establecimiento de modelos de calidad. Se utilizó a 372 profesionales como muestra de estudio, que permitieron determinar las diferentes habilidades $y$ destrezas que debe manejar el líder en la actualidad. Las competencias gerenciales que deben tener los directivos de las empresas son 1) el manejo de dirección, necesaria para el emprendimiento de procesos de gestión administrativa; 2) el liderazgo, como una actuación de compromiso, iniciativa, creatividad, visión de futuro y sólidos valores en el desempeño personal y profesional; y 3) la gestión del cambio, a través de actividades y proyectos innovadores para alcanzar las metas con relación a su competencia en el mercado.

\footnotetext{
1 Docente de la Universidad Técnica de Ambato. Ecuador

2 Docente de la Universidad Técnica de Ambato. Ecuador

3 Docente de la Universidad Técnica de Ambato. Ecuador

4 Docente de la Universidad Técnica de Ambato. Ecuador

5 Docente de la Universidad Técnica de Ambato. Ecuador

6 BH Consultores. Ecuador
}

Revista científica Ciencia y Tecnología Vol 18 No 17 págs. 205-217 http://cienciaytecnologia.uteg.edu.ec 
Palabras clave: Habilidades gerenciales, sectores productivos, competencias, manejo de conflictos

\begin{abstract}
The purpose of this study is to perform a prospective analysis that facilitates the construction and development of managerial skills through a model that reflects a profile by management skills for managers of the productive sectors of the province of Tungurahua in the context of continuous improvement and establishment of quality models. 372 professionals were used as a study sample, which allowed Determine the different skills and abilities that the leader must handle today. The managerial competencies that the directors of the companies should have are: 1 ) management, necessary for the undertaking of administrative management processes; 2) leadership, as a performance of commitment, initiative, creativity, vision of the future and solid values in personal and professional performance; and 3 ) change management, through innovative activities and projects to achieve the goals in relation to their market competition.
\end{abstract}

Keywords: Management skills, productive sectors, competencies, conflict management

Este artículo, forma parte de los resultados del proyecto de investigación institucional: "LAS HABILIDADES GERENCIALES DE LOS SECTORES PRODUCTIVOS DE TUNGURAHUA Y EL CUMPLIMIENTO EN EL APORTE AL PLAN NACIONAL DEL BUEN VIVIR", presentado en la Dirección de Investigación y Desarrollo DIDE, de la Universidad Técnica de Ambato y aprobado según resolución de Consejo Universitario: 1576-CU-P-2017, del 08 de agosto de 2017 y que finalizará su ejecución, el 22 de noviembre de 2018.

\title{
Introducción
}

Actualmente el constante crecimiento empresarial a nivel mundial solicita que el perfil del gerente adquiera y este compuesto de diversos conocimientos, una diversidad en las habilidades y con una capacidad de liderazgo. Blackler, Reed, y Whitaker (1993) afirma que la gerencia es considerada como una actividad social dirigida a la organización y dirección de las empresas que se encamina bajo un objetivo primordial, el cual es la obtención de resultados a través de criterios de eficiencia, eficacia y efectividad.

La relación de las habilidades gerenciales con los sectores productivos flexibiliza a los gerentes a establecer estrategias para mantener y elevar el desarrollo de las ventajas competitivas de las organizaciones. El crecimiento de las empresas hoy en día, requiere de gerentes con habilidades y destrezas que les permita ser competitivos, mejorar relaciones con colaboradores proveedores, clientes y todas aquellas partes que intervengan en la cadena de valor (Ruiz Dalgo, Bonilla Jurado y Masaquiza Caiza, 2018). La innovación es un reto que exige el desarrollo de nuevos conocimientos que potencializara las competencias necesarias que se requiere para 
la gestión de la gerencia ante los cambios.

Las habilidades gerenciales son capacidades con las cuales un líder debe contar para desempeñar los deberes y actividades inherentes a su cargo. Este proceso permitirá determinar el rol futuro del gerente con base a posibles escenarios para desplegar acciones conjugando los conocimientos, la competencia y la conectividad de tal manera que se pueda potencializar el desarrollo de los sectores productivos.

El propósito de este estudio es realizar un análisis prospectivo que facilita la construcción y desarrollo de las habilidades gerenciales a través de un modelo que refleja un perfil por competencias gerenciales para los gerentes de los sectores productivos de la provincia de Tungurahua en el contexto de la mejora continua y establecimiento de modelos de calidad. I CIMES 2018 Miami - Florida Número especial.

\section{Desarrollo}

Como desarrollo de la investigación, se determina un marco referencial con teorías que aportan al desarrollo metodológico.

\section{La gerencia en la Pyme y su desarrollo económico}

Al hacer énfasis en el perfil gerencial, se observa que las empresas y hasta el mismo individuo deben seguir una serie de procesos, adquirir habilidades y destrezas para lograr alcanzar el éxito deseado. En tal sentido, para Eunamet (2006) la tendencia postmoderna en el terreno gerencial puntualiza en la relatividad de cualquier visión o postura conceptual. Además de resaltar el papel de la subjetividad de la variable cultural en cualquier análisis de lo organizativogerencial. Por tanto, si las Pyme quiere desempeñar un rol significativo en pro de la economía del país, debe contar con una gerencia capaz de interpretar su entorno, diagnosticar e interpretar la realidad, así como saber enfrentar la competitividad con ventajas competitivas favorecedoras, creando así una cultura proactiva y humanista en la empresa.

\section{Habilidades gerenciales}

De acuerdo a la definición de Uslay, Morgan y Sheth (2009) afirma que la gerencia se considera como una actividad social orientada a la dirección de las organizaciones, tiene como objetivo primordial la obtención de resultados bajo criterios de eficiencia, eficacia y efectividad. Las habilidades gerenciales son capacidades y destrezas con las cuales un líder debe contar para desempeñar los deberes y actividades inherentes a su cargo.

Por otra parte, Blackler y otros (1993) caracteriza las habilidades como una combinación de conocimientos, destrezas, comportamientos y actitudes que necesita un gerente para ser eficaz. Si el emprendedor cuenta con más habilidades, el costo de oportunidad de la coordinación de trabajadores, en términos productivos, es mayor, así trabajadores calificados deberían necesitar menos 


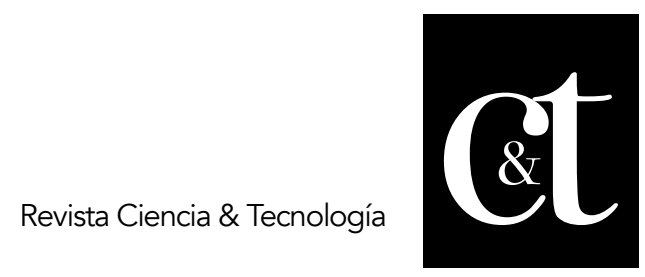

No. 17, 31 de enero de 2018

ISSN impreso: 1390 - 6321

atención del empresario (Rodríguez Moreno \& Gómez Murillo, 2014).

Modelo prospectivo de Habilidades Gerenciales

En el entorno empresarial se requiere que los gerentes contengan habilidades y destrezas que faciliten que sus colaboradores desarrollen ambientes de colaboración, motivación y compromiso, contribuyendo al crecimiento del personal que a su vez se reflejara en la obtención de mejores resultados en el desempeño del mismo (Whetten \& Cameron, 2005).

Los emprendedores poseen un perfil de éxito o "perfil emprendedor", alrededor del cual muchos autores han tratado de agrupar rasgos, características, atributos, entre otros, que parecieran ser los más significativos, ponderando y diseñando cuestionarios para determinar si una persona posee lo que se requiere para emprender, obviando entonces el enfoque de competencias. Además, se sugieren tipologías de emprendedor, sin que se defina con claridad un concepto sobre el mismo (Barreto y Thais, 2015).

Se ha determinado que la administración a través de las habilidades es un factor determinante en el éxito empresarial dejando ver que para direccionar a las organizaciones al éxito se requiere directivos que desarrollen sus competencias y habilidades, demuestren habilidades paradójicas. Esto quiere decir, que los directivos más eficaces son tanto participativos como impulsores tanto fomentadores como competitivos. Sus rasgos de flexibilidad y creatividad son estables y racionales (Whetten \& Cameron, 2005).

A su vez Naranjo (2014) afirma en un estudio realizado a las pequeñas y medianas empresas (PYMES) que su representación y contribución al PIB es del 50\%, con relación a la estructura empresarial establecido en un modelo de competencias que garantizan el éxito y competitividad, ya que las competencias esenciales apuntalan los procesos de la empresa generando un valor añadido y son la clave del éxito. I CIMES 2018 Miami - Florida Número especial. 
Tabla 1. Habilidades de un administrador

\begin{tabular}{|c|c|}
\hline Habilidades estratégicas & $\begin{array}{c}\text { Habilidades con relación a las } \\
\text { tareas }\end{array}$ \\
\hline $\begin{array}{l}\text { - Valoración del entomo } \\
\text { - Desarrollo de la estrategia } \\
\text { - Trazar la estrategia y definir la misión } \\
\text { - Implantar la estrategia } \\
\text { - Congruencia con los recursos } \\
\text { humanos }\end{array}$ & $\begin{array}{l}\text { - Establecer y lerarquizar los } \\
\text { objetivos } \\
\text { - Desarrollar un plan de acción e } \\
\text { implantarlo } \\
\text { - Responder de una manera flexible } \\
\text { - Crear valor } \\
\text { - Asignación de los recursos } \\
\text { humanos } \\
\text { - Gestionar eficientemente }\end{array}$ \\
\hline \multicolumn{2}{|c|}{ Habilidades con las personas } \\
\hline $\begin{array}{l}\text { - Delegación } \\
\text { - Influencia } \\
\text { - Motivación } \\
\text { - Manejo del conflicto } \\
\text { - Ganar una negociación }\end{array}$ & $\begin{array}{l}\text { - Redes } \\
\text { - Presentacion } \\
\text { - Comunicación no verbal } \\
\text { - Escuchar } \\
\text { - Gestion cross-cultural } \\
\text { - Equipo de trabajo heterogéneo }\end{array}$ \\
\hline
\end{tabular}

Elaborado por: Autores

De esta forma Medina Lorza y Castañeda Zapata (2010) estipulan un perfil de competencias dentro de las habilidades gerenciales que debe poseer un gerente 0 director dentro de su organización productiva. Uno de los primeros en mencionar, es el liderazgo representado en el manejo de las relaciones interpersonales, su rol consiste en la motivación y estimulación en la participación de sus miembros que conforman la empresa generándoles compromiso en las distintas áreas de trabajo tanto administrativas como productivas.

Según Jiménez, Ocegueda, García y Guzmán (2013) indica que las funciones básicas de un administrador requieren de un conjunto de habilidades para ejecutarse correctamente ya que la mayoría de las actividades directivas son únicas y no constan de una mejor alternativa para realizarlas. I CIMES 2018 Miami Florida Número especial.

A continuación, se describe las habilidades directivas para el éxito empresarial. Gestión del tiempo Inteligencia emocional Toma de decisiones Capacidad de 


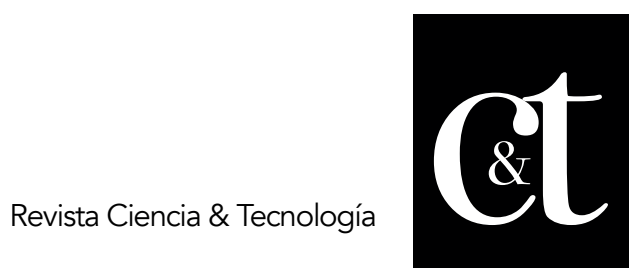

No. 17, 31 de enero de 2018

ISSN impreso: 1390 - 6321

aprender Desarrollo de la visión Estrategia y desarrollo personal.

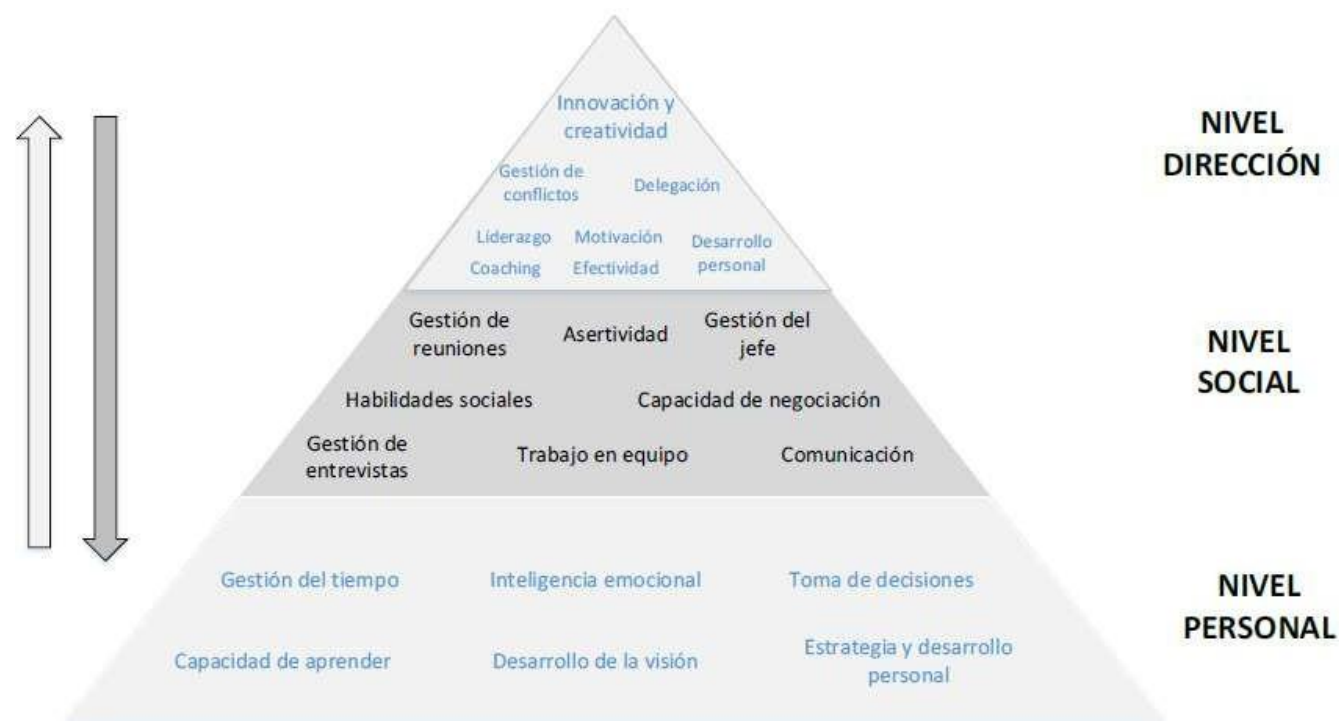

Gráfico 1. Pirámide de habilidades gerenciales

Como lo determina el gráfico 1 el análisis de la literatura sobre las habilidades gerenciales nos ha permitido desarrollar una clasificación tomando en cuenta las habilidades gerenciales que proponen Whetten y Cameron (2005) a su vez basado en el modelo de pirámide de habilidades gerenciales de Pereda, López-Guzmán y González (2014) determina innovación y creatividad del nivel de dirección y la capacidad de negociación del nivel social estableciendo la propuesta de las habilidades que serán analizadas en cada uno de los gerentes de los sectores productivos de Tungurahua.

Tabla 2. Habilidades gerenciales en los sectores productivos

\begin{tabular}{|c|c|c|c|}
\hline PERSONALES & INTERPERSONALES & GRUPALES & COMPLEMENTO \\
\hline $\begin{array}{c}\text { Solución analítica } \\
\text { y creativa de } \\
\text { problemas }\end{array}$ & Manejo de conflictos & $\begin{array}{c}\text { Facultamiento } \\
\text { y delegación }\end{array}$ & $\begin{array}{c}\text { Innovación y } \\
\text { creatividad }\end{array}$ \\
\hline Manejo de estrés & $\begin{array}{c}\text { Motivación de los } \\
\text { empleados }\end{array}$ & $\begin{array}{c}\text { Formación de } \\
\text { equipos } \\
\text { eficaces }\end{array}$ & $\begin{array}{c}\text { Capacidad de } \\
\text { negociación }\end{array}$ \\
\hline & Comunicación de apoyo & & Liderazgo \\
\hline & Ganar poder e influencia & & \\
\hline
\end{tabular}

Elaborado por: Autores 


\section{Metodología}

El presente trabajo se elaboró bajo un enfoque positivista a través de este estudio se busca obtener un conocimiento de las variables planteadas con base a la realidad mediante teorías, técnicas y métodos en cuanto a las habilidades gerenciales nos permitirá obtener respuestas validas a las interrogantes que ha planteado desde el inicio.

La investigación tiene un enfoque cualitativo, descriptivo ya que está orientada a caracterizar, analizar e interpretar las habilidades gerenciales que serán fundamentales para la dirección empresarial determinadas a través de un análisis prospectivo en los sectores productivos de la provincia de Tungurahua.

La recopilación de información se realizó mediante una investigación de campo, a través de la aplicación de una encuesta a los directivos de las distintas empresas en cada uno de los sectores productivos diseñada para la identificación de las habilidades y destrezas gerenciales que se aplican principalmente en la organización.

Se procesó la información recopilada a través de la encuesta en el programa estadístico SPSS posteriormente los datos obtenidos fueron ingresados en el programa Mic Mac con el objetivo de identificar las variables influyentes y dependientes y por ende aquellas esenciales.

Población y muestra

A continuación, se detalla la muestra de estudio con la cual se trabajó la investigación para que pueda ser recolectada la información.

\section{Tabla 3. Muestra de Estudio}

\begin{tabular}{|c|c|c|c|}
\hline SECTORES & $\%$ & & MUESTRA \\
\hline Alimentos & 32 & 7840 & 119 \\
\hline Cadena agroforestal & 2 & 490 & 7 \\
\hline Cuero y calzado & 15 & 3675 & 56 \\
\hline Farmacéutico & 4 & 980 & 15 \\
\hline Turismo & 6 & 1470 & 22 \\
\hline Petroquímico & 10 & 2450 & 37 \\
\hline Tecnología & 4 & 980 & 15 \\
\hline Textil & 14 & 3430 & 53 \\
\hline Metalmecánica & 13 & 3185 & 48 \\
\hline & 100 & 24500 & 372 \\
\hline
\end{tabular}

Elaborado por: Autores 


\section{Resultados y Discusión}

En cuanto al aporte al desarrollo personal y profesional del perfil gerencial de la provincia de Tungurahua, se incluye el impacto y desarrollo que generan en ellos a través de los libros analizados, marcando una diferencia o puntualizando una diferencia entre el desarrollo personal y el desarrollo profesional, asimilando el personal con los procesos motivacionales, de autoestima, autoconcepto y el profesional con los de la autoeficacia como lo establece el gráfico 2.

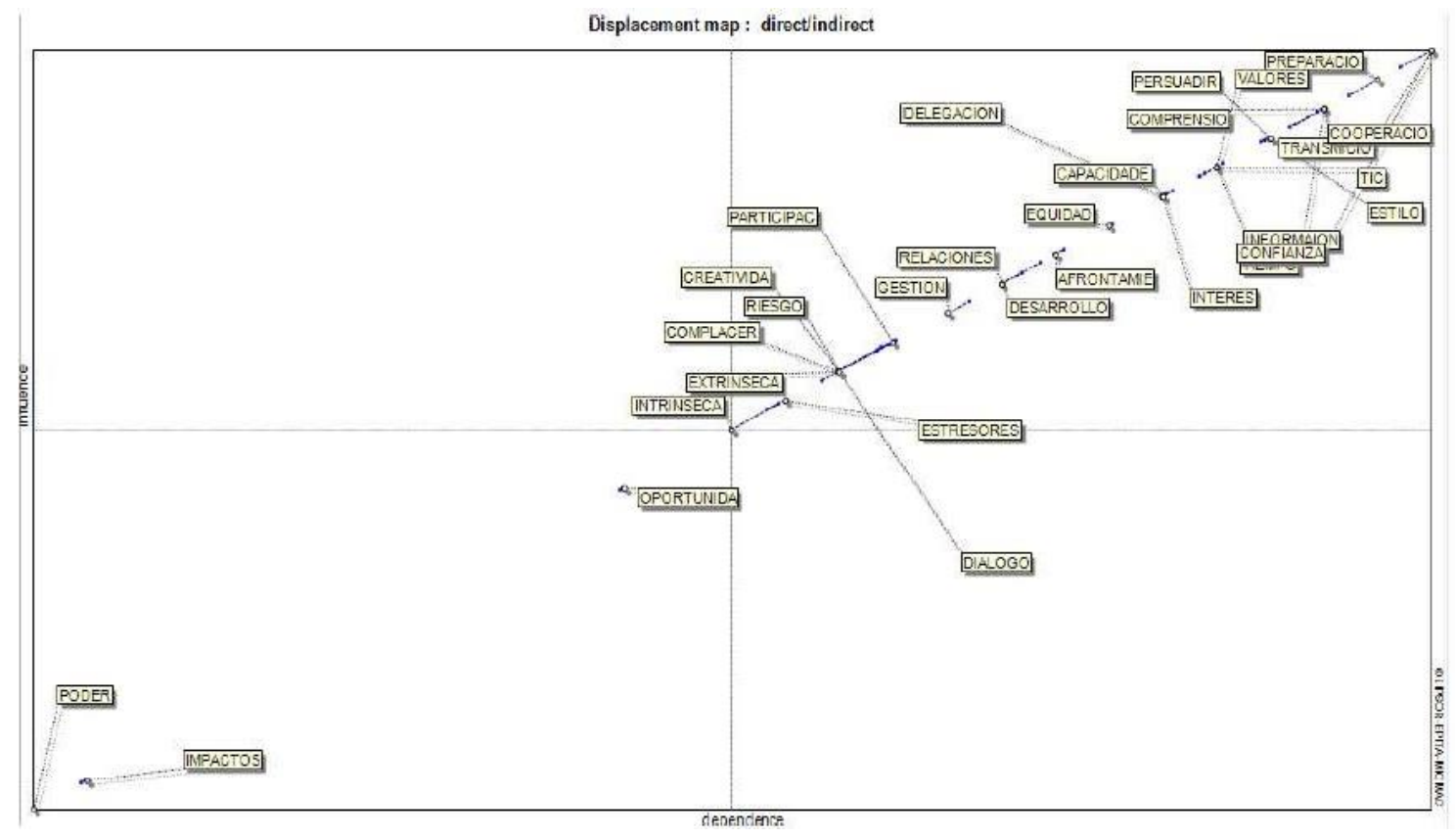

\section{Gráfico 2. Perfil gerencial estándar para los sectores productivos de la provincia de Tungurahua}

\section{Análisis General}

Primer cuadrante (Influencia Alta): Refleja los factores que influyen directamente en el perfil de las habilidades del Gerente dentro de los sectores productivos, por lo tanto, los empresarios deberán requerir de estas habilidades para el desarrollo eficaz de su organización.

Motivación de empleados - información

El nivel gerencial para que pueda motivar a los empleados necesita estar informado y abierto a la educación, se puede argumentar que uno de los poderes de incentivo más importantes es dejar que los empleados se mejoren a sí mismos, el éxito de los empleados está conectado a ser administrados de la manera correcta y motivados al compromiso, eficiencia y productividad. 


\section{Liderazgo - transmisión}

Una habilidad de importancia donde los lideres comprende la complejidad y visión de las situaciones, con el fin de construir decisiones que les permitan aprender de sus vivencias y ser creativos e innovadores al momento de resolver los problemas, un líder debe aprender a guiar y a transmitir a sus colaboradores ideas que permitan hacerles crecer para el beneficio de la organización.

Manejo de estrés - preparación

Una habilidad que debe ser esencial para el manejo de este problema ya que el estrés se origina por una serie de situaciones negativas que pueden ser problemas de salud, familia, trabajo entre otras todas estas situaciones son generadores de estresores. También, son las personas las encargadas de generar estrés al no encontrarse preparadas para afrontar este tipo de situaciones.

Ganar poder e influencia - confianza

Cuando los líderes gestionan activamente las emociones de los seguidores a través de estrategias de gestión de emociones personales, tales conductas de líder se convierten en una pieza integral de información que los utilizan para evaluar, intercambiar y mantener esa relación con sus líderes y de esa manera general una confianza dentro de la empresa.

Liderazgo - comprensión

Aquí el líder hace las veces de un maestro, guía o facilitador responsable, orientado a que los colaboradores comprendan la complejidad y visión de las situaciones, con el fin de construir decisiones que les permitan aprender de sus vivencias y ser creativos e innovadores al momento de resolver los problemas.

Comunicación de apoyo - estilo

Una habilidad de gran importancia mediante el uso del humor en la comunicación permite crear ambientes más agradables y favorecer las relaciones grupales, aquellas personas que tienen un estilo de comunicación orientado al humor muestran mayor flexibilidad. Se ha demostrado que aquellos jefes y supervisores que se expresan con un cierto grado de humor son vistos como más efectivos.

Ganar poder e influencia - persuadir

Cuando los seguidores hacen atribuciones positivas sobre el líder y sienten una sensación de obligación basada en expectativas, se crea un sentido de obligación a través del comportamiento de líder solidario de reformular las molestias del lugar de trabajo. Cuando los líderes gestionan activamente las emociones de los seguidores a través de estrategias de gestión de emociones personales, tales conductas de líder se convierten en una pieza integral de información que los seguidores utilizan para evaluar, intercambiar y mantener esa relación con sus líderes. 
Motivación de los empleados - valores

La motivación como la participación y el compromiso activo de una persona para lograr los resultados prescritos. Con esto se puede decir que, la motivación es fundamental para que las organizaciones funcionen de manera correcta. Si no existe motivación los empleados no pueden ofrecer lo mejor de sí mismos y el rendimiento de la empresa es menos eficiente, aporta que la motivación es la estrategia de asignar a los ejecutivos que son capaces de estimular la motivación de los empleados, en otras palabras, los líderes, para el personal administrativo es una estrategia efectiva en la gestión de la motivación.

Capacidad de negociación

Para la realización de una negociación, un líder se basa en un proceso de negociación que pueden desarrollarse haciendo uso de las tecnologías de información TIC's con una intensidad y frecuencia que dependerá de las necesidades y preferencias de la organización. No obstante, este tipo de herramientas mejoran considerablemente las relaciones comerciales en un tiempo considerable de las organizaciones que realizan procesos de importación y/o exportación.

Solución analítica y creativa de problemas - intereses

La habilidad del nivel gerencial se basa en el aprendizaje que poseen al buscar soluciones creativas, soluciones a fondo para que de la misma manera se solucione el problema desde la raíz y no vuelva a ocurrir, ya que de lo contrario sino se empieza por cambiar la manera de solucionar los problemas seguirán ocurriendo varias veces. La creatividad es uno de los elementos más importantes que tiene el hombre, para mejorar de manera efectiva el desarrollo de sus habilidades directivas, y la solución de problemas en todas las etapas de su vida social y profesional.

Formulación de equipos eficaces

La conformación de los equipos de trabajo se basa en las habilidades comprendidas por cada colaborador para hacer frente al alcance de los objetivos en común, desarrollar tareas conjuntas, ayudar a aumentar la calidad de trabajo, evitar obstáculos, innovar, atraer recursos, generar oportunidades y explorar las posibilidades en el cumplimiento de las metas u objetivos organizacionales.

\section{Modelo de habilidades gerenciales}

El modelo de habilidades gerenciales parte del análisis prospectivo de las industrias, de lo cual se obtienen las principales características que forman parte del perfil gerencial que requieren las empresas de la provincia de Tungurahua. 


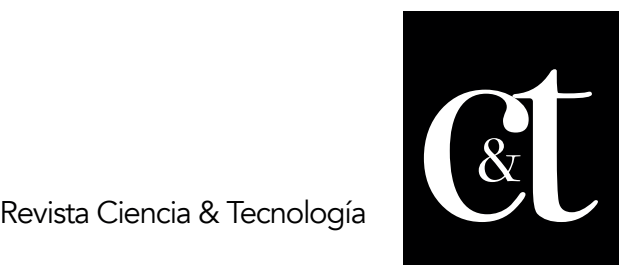

No. 17, 31 de enero de 2018

ISSN impreso: 1390 - 6321

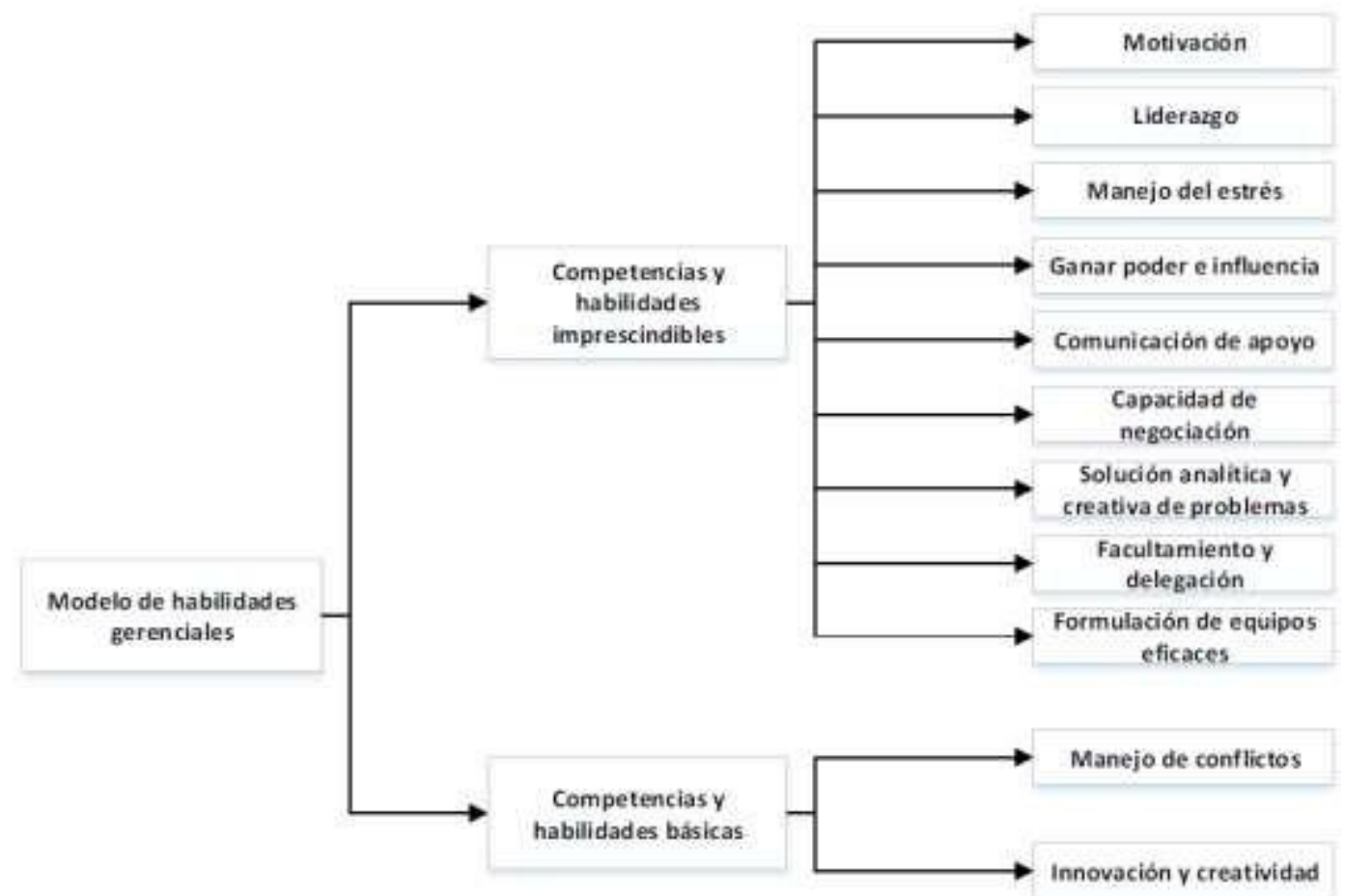

\section{Gráfico 3. Perfil gerencial estándar para los sectores productivos de la provincia de Tungurahua}

Dentro del análisis prospectivo se establece las habilidades que se forman mediante capacitaciones como el manejo del estrés, manejo de conflictos, comunicación de apoyo, innovación y creatividad y la formación de equipos eficaces son habilidades que son desarrolladas a través de cursos de capacitación que son otorgados con la finalidad de preparar al nivel gerencial ante la prospectiva a la cual se va a tener que enfrentar.

Las habilidades gerenciales que se adquieren a través de una formación académica podemos destacar la solución analítica y creativa de problemas, motivación de empleados, ganar poder e influencia, facultamiento y delegación, capacidad de negociación y liderazgo, habilidades obtenidas mediante un aprendizaje consecuente en el cual se adquiere conocimientos profundos de cada habilidad para ser desarrollada dentro de cada organización con la finalidad de que contribuya a una mejora en la empresa.

\section{Conclusiones}

Se identifica bajo la aplicación de la encuesta a directivos de distintas empresas en los sectores productivos de Tungurahua y análisis prospectivo aquellas habilidades primordiales con las cuales los gerentes deberán trabajar a lo largo de su gestión directiva identificando la diferencia en cada uno de los sectores. 
Se conceptualiza las habilidades personales, interpersonales y grupales, de esta manera se establece un modelo de habilidades gerenciales basado en distintos aportes científicos. Se analiza cada una de las habilidades gerenciales que aplican los sectores productivos, a través de la identificación del factor principal en los diferentes sectores "motivación a los empleados". El manejo del estrés es una de las principales habilidades aplicadas en los diferentes sectores productivos, así como la innovación y la creatividad, la solución analítica y creativa de problemas. Que se encuentra presente como primordial en todos los sectores.

La prospectiva ha permitido determinar las habilidades esenciales que permiten la gestión de los directivos en cada uno de los sectores, identificando también aquellas no aplicadas o con poca relevancia según el sector.

\section{Referencias Bibliográficas}

Barreto, N. y Thais, T. (2015). Perfil del emprendedor rural propuesta de enfoque de competencias. Anales, 34.

Blackler, F., Reed, M., \& Whitaker, A. (1993). Editorial introduction: knowledge workers and contemporary organizations. Journal of Management Studies, 30(6), 851-862. https://doi.org/10.1177/0149206310385697

Jiménez, C., Ocegueda, C., García, I. y Guzmán, I. (2013). Perfil del administrador de recursos humanos y desempeño organizacional en el sector industrial de $\mathrm{H}$. Matamoros. MEMORIAS ARBITRADAS DEL VIII CONGRESO DE INGENIERÍA INDUSTRIAL Y DE SISTEMAS FACULTAD DE INGENIERIA MECANICA Y ELECTRICA. Recuperado a partir de http://eprints.uanl.mx/8572/1/PERFIL DEL ADMINISTRADOR.pdf

Knight, G. (2000). Entrepreneurship and marketing strategy: The SME under Globalization. Journal of International Marketing, Vol. 8, No. 2 (2000), pp. 1232.

Medina Lorza, A. F. y Castañeda Zapata, D. I. (2010). Competencias Requeridas en los encargados de recursos humanos para el desempeño eficaz de empresas industriales de Cali, Colombia. Estudios gerenciales, 26(115), 117-140. Recuperado a partir de http://www.scielo.org.co/scielo.php?script=sci_arttext\&pid=S012359232010000200007

Naranjo, R. (2014). Habilidades gerenciales en los líderes de las medianas empresas de Colombia. Pensamiento y Gestión, 73(15), 145. https://doi.org/10.14482/pege.38.7703

Pereda, F.J., López-Guzmán, T. y González, F. (2014). Las habilidades directivas como ventaja competitiva. El caso del sector público de la provincia de Córdoba (España). Intangible Capital, vol. 10, núm. 3, p. 528-561.

Rodríguez Moreno, D., y Gómez Murillo, A. (2014). Las competencias emprendedoras en el departamento de Boyacá. Apuntes del CENES, 27. 


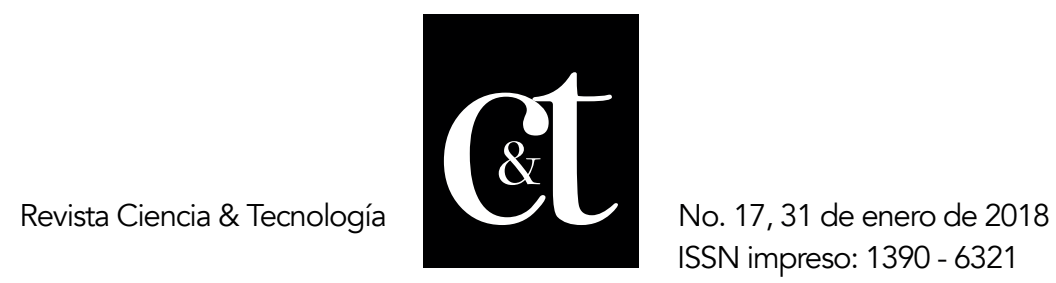

Ruiz Dalgo, D. V., Bonilla Jurado, D. M., y Masaquiza Caiza, C. S. (2018). Ajuste estratégico en la cadena de suministros para la creación de valor de la marca Jean Up. Universidad y Sociedad, Revista Científica de la Universidad de Cienfuegos, 10(1), 25-32. Recuperado a partir de https://rus.ucf.edu.cu/index.php/rus/article/download/757/858/

Uslay, C., Morgan, R. E., \& Sheth, J. N. (2009). Peter Drucker on marketing: An exploration of five tenets. Journal of the Academy of Marketing Science, 37(1), 47-60. https://doi.org/10.1007/s11747-008-0099-8

Whetten, A., \& Cameron, S. (2005). Desarrollo de Habilidades Directivas. Mexico: Pearson Educacion. 